\title{
T-ray tomographic imaging
}

\author{
Bradley Ferguson ${ }^{a, b, c, d}$, Shaohong $\mathrm{Wang}^{a}$, Doug $\mathrm{Gray}^{c, d}$, Derek Abbott ${ }^{b, c}$ and X.-C. Zhang ${ }^{a}$ \\ ${ }^{a}$ Center for Subsurface Sensing and Imaging Systems, \\ Rensselaer Polytechnic Institute, Troy, NY 12180, USA \\ ${ }^{b}$ Centre for Biomedical Engineering, The University of Adelaide, SA 5005, Australia \\ ${ }^{c}$ Department of Electrical \& Electronic Engineering, The University of Adelaide, SA 5005, \\ Australia \\ ${ }^{d}$ CRC for Sensor, Signal and Information Processing, \\ Technology Park, Mawson Lakes Boulevard, Mawson Lakes, SA 5095, Australia
}

\begin{abstract}
We present three T-ray (terahertz wave) tomographic imaging modalities: T-ray computed tomography, T-ray diffraction tomography, and tomographic imaging with a Fresnel binary lens. Each of these techniques uses pulses of broadband terahertz radiation to obtain 3-dimensional images of targets with wide potential application. We present images demonstrating the performance of each technique and discuss their relative advantages.
\end{abstract}

Keywords: Terahertz imaging, tomography, diffraction, Fresnel lens

\section{INTRODUCTION}

There has been considerable recent interest in tomographic methods of THz imaging. ${ }^{1-5}$ These methods extend the advantages of 2-dimensional T-ray imaging ${ }^{6}$ to applications involving 3-dimensional (3D) targets. Short pulses of broadband $\mathrm{THz}$ radiation (with typical frequency components ranging from $100 \mathrm{GHz}$ to $3 \mathrm{THz}$ ), are used to illuminate the target. Coherent detection methods are used to allow the reflected or transmitted $\mathrm{THz}$ pulse profile to be measured. This provides spectral information over a broad range in the important far-infrared band. In spectroscopy applications this information has been used for semiconductor characterization, ${ }^{7}$ the identification of gas mixtures ${ }^{8}$ and label-free DNA analysis. ${ }^{9}$ A further advantage of this frequency range is the fact that many common materials including cloth, paper, plastics and cardboard are relatively non-absorbing. 3D T-ray tomography systems therefore have a large number of potential applications. The first demonstrated T-ray tomography system ${ }^{1}$ was based on the principles of reflection-mode ultrasound. This technique is capable of resolving the $3 \mathrm{D}$ refractive index profile for objects consisting of well-separated layers of differing refractive index. While this technique provides extremely sensitive depth resolution of the order of $1 \mathrm{~mm}$, the current reconstruction algorithms are only applicable given a number of assumptions which restrict its applicability. These algorithms neglect multiple reflections, absorption and dispersion in the object to be imaged. These restrictions have motivated research into more general tomographic imaging methodologies. We have recently reported 3 novel techniques for transmission mode tomography using $\mathrm{THz}$ radiation. Here we describe these methods in detail and discuss the applicability of each. Section 2 presents the 3 tomographic modalities: T-ray computed tomography (T-ray CT), T-ray diffraction tomography (T-ray DT) and Fresnel lens tomography. In Section 3 the techniques are compared and their relative advantages discussed. The conclusions and future work are outlined in Section 4.

Further author information: (XCZ) E-mail: zhangxc@rpi.edu, Telephone: 15182763079 ,

(DA) E-mail: dabbott@eleceng.adelaide.edu.au, 


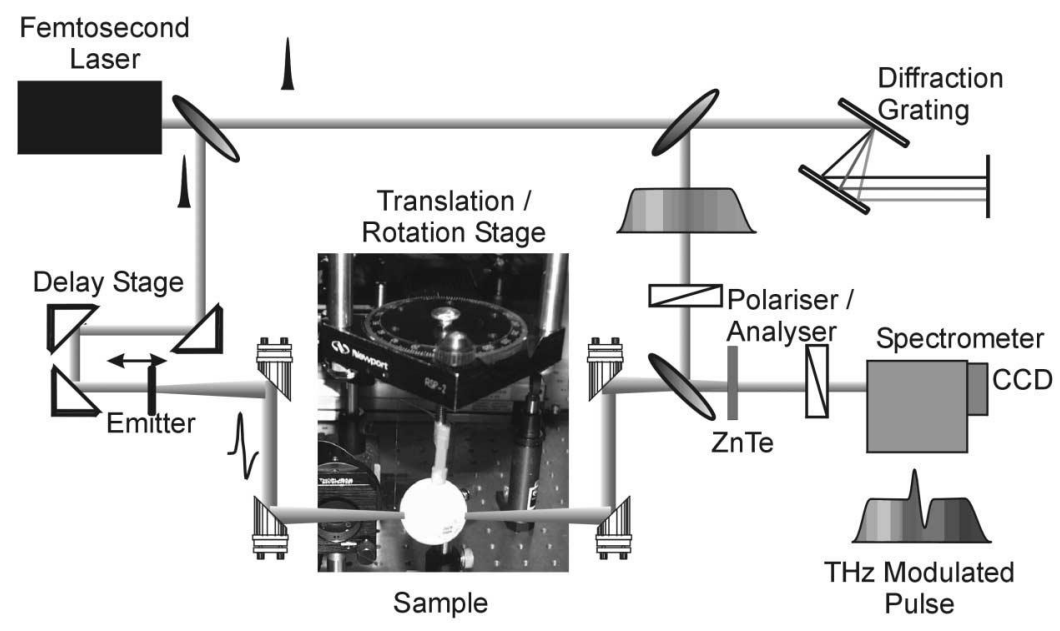

Figure 1. Simplified hardware schematic used for T-ray CT. The ultrafast laser pulses are split into pump and probe beams. The pump beam triggers a biased $(2000 \mathrm{~V})$ wide aperture antenna to generate $\mathrm{THz}$ pulses which are focused on the target using parabolic mirrors. The probe beam is chirped using a grating pair to a pulse width of $30 \mathrm{ps}$. The THz temporal profile is encoded on the probe pulse using the $4 \mathrm{~mm}$ thick ZnTe electro-optic detector crystal and a pair of crossed polarisers. A spectrometer and CCD camera are used to recover the THz signal. Inset: a photo of the rotation stage and sample is shown. The sample is a dielectric sphere.

\section{TOMOGRAPHY TECHNIQUES}

\subsection{T-ray CT}

The image formation model and the reconstruction algorithms employed in T-ray CT are based on those of x-ray CT. Figure 1 shows the schematic of the T-ray CT system. T-rays are focused, transmitted through the target and detected. The target is then raster scanned and rotated. The hardware is a simple extension of the chirped probe beam $\mathrm{THz}$ imaging system.

THz pulses are generated using a regeneratively amplified Ti:sapphire laser (Spectra Physics Hurricane) with an average output power of $700 \mathrm{~mW}$, center wavelength of $800 \mathrm{~nm}$, a pulse duration of $130 \mathrm{fs}$ and a repetition rate of $1 \mathrm{kHz}$, incident on a $14 \mathrm{~mm}$ wide aperture photoconductive antenna with a bias of $2000 \mathrm{~V}$. A wide aperture antenna is used with a loosely focused pump beam to allow the very high pump power available from the Hurricane to be used without damaging the emitter. The $\mathrm{THz}$ radiation is focused using off axis parabolic mirrors to a spot size of approximately $1 \mathrm{~mm}$ on the target. The transmitted $\mathrm{THz}$ pulse is collected using parabolic mirrors and focused onto a $4 \mathrm{~mm}$ thick $<110>$ ZnTe electro-optic (EO) detector crystal. The acquisition speed of the data is an important concern. We use a linearly chirped optical probe beam for electro-optic detection of the $\mathrm{THz}$ pulses. ${ }^{10}$ Using this technique the full $\mathrm{THz}$ waveform is measured simultaneously, dramatically accelerating the imaging speed. The optical probe pulse is linearly chirped and temporally stretched to a pulse width of $30 \mathrm{ps,}$ using a grating pair. The $\mathrm{THz}$ pulse modulates the probe pulse via the $\mathrm{EO}$ effect and is recovered using the spectrometer and CCD camera. Using a CCD exposure time of $50 \mathrm{~ms}$ the signal-to-noise ratio (SNR) for the system was approximately 100. The exposure time may be reduced to $5 \mathrm{~ms}$ at the expense of SNR. The sample is mounted on a X-Y translation stage and raster scanned to acquire an image. It is then rotated and the $\mathrm{THz}$ image recorded at each projection angle.

The filtered backprojection algorithm is widely used for the reconstruction of x-ray CT images. ${ }^{11}$ This method is based on a line integral model. To formulate the reconstruction algorithm for T-ray CT we assume that the detected $\mathrm{THz}$ signal is approximately related to the transmitted pulse by a line integral of the form 


$$
P_{d}(\omega, \theta, l)=P_{i}(\omega) \exp \left[\int_{L(\theta, l)} \frac{-i \omega \hat{n}(r)}{c} d r\right]
$$

where $P_{d}$ is the Fourier transform of the detected THz signal at a frequency $\omega$, a projection angle $\theta$ and a horizontal offset from the axis of rotation $l . P_{i}$ is the Fourier component of the incident THz signal at the same frequency, $c$ is the speed of light, $L$ is the straight line between the source and detector and $\hat{n}(r)=n(r)+i k(r)$ is the unknown complex refractive index of the sample.

T-ray CT allows the reconstruction of both the absorption coefficient $k$ and the real refractive index $n$ of the sample at all available frequencies by extracting different information from the measured data as input to the filtered backprojection algorithm. For example, to perform the reconstruction for the real refractive index $n(\omega)$ at a given frequency, we use

$$
s(\theta, l)=\arg \left[\frac{P_{d}(\omega, \theta, l)}{P_{t}(\omega, \theta, l)}\right] .
$$

The filtered backprojection algorithm involves computing the Fourier transform of the projection data, $s(\theta, l)$, to yield $S(\theta, w)$, where $w$ is the spatial frequency in the $l$ dimension. The filtered backprojection algorithm can be described by the following equation:

$$
\hat{n}(x, y)=\int_{0}^{\pi}\left[\int_{-\infty}^{\infty} S(\theta, w)|w| \exp [i 2 \pi w l] d w\right] d \theta
$$

where $l=x \cos \theta+y \sin \theta$. The refractive index is then recovered by $n(x, y, w)=1+(s(x, y) c) /(w d r)$, where $d r$ is the reconstruction grid size.

T-ray CT has the potential to identify targets based on their frequency response in the far-infrared. Figure 3 shows the reconstruction of a simple sample (shown in Fig. 2), consisting of a sheet of polyethylene bent into an 'S' shape, using the data at 4 different frequencies. A coarse sampling step of $1 \mathrm{~mm}$ in the $z$ and $l$ dimensions was used to obtain projection images for each $10^{\circ}$ angular increment. The resolution of the reconstruction improves as the frequency increases however the SNR decreases because most of the source power is at frequencies below $0.5 \mathrm{THz}$. Therefore the reconstructions at higher frequencies suffer from additional artifacts caused by noise. A 3D image can be formed by reconstructing each horizontal slice and surface rendering them as illustrated in Fig. 4 .

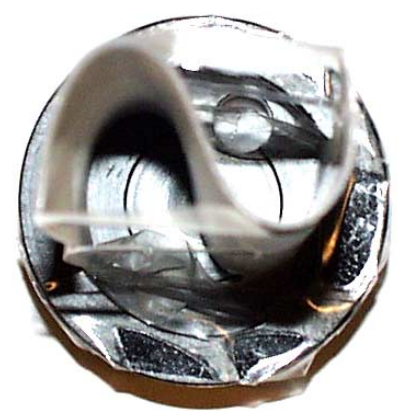

Figure 2. Top view of polyethylene sheet folded into an 'S' shape. The polyethylene was held in place using scotch tape. The sample was mounted such that the THz beam was in the plane of the paper and the sample was rotated about the $\mathrm{z}$-axis (the axis pointing out of the page). 

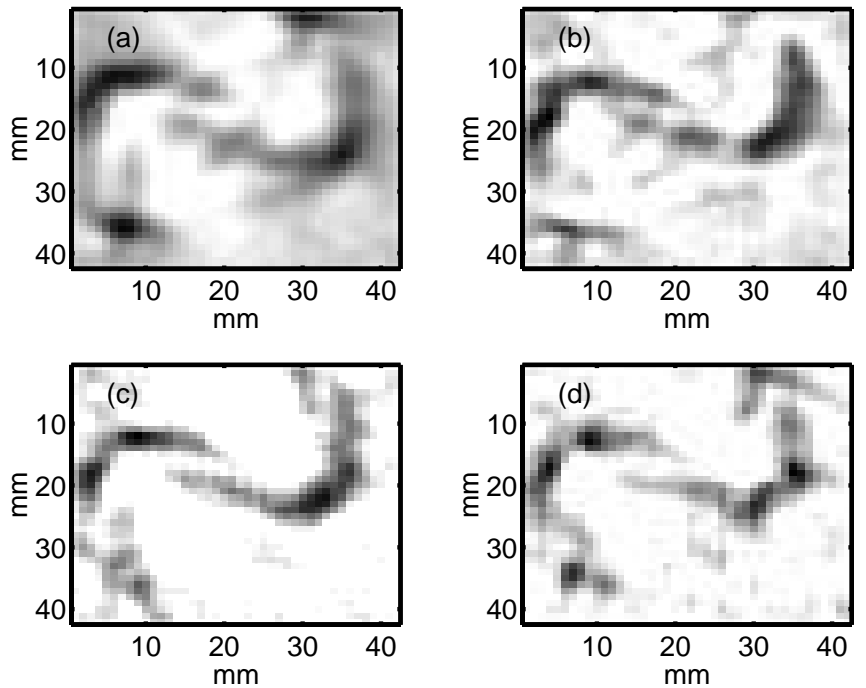

Figure 3. Frequency dependent reconstructions of the sheet of polyethylene. The measured data was Fourier transformed and the phase of the Fourier domain responses at 4 different frequencies was used to reconstruct the sample. Reconstructed cross sectional slices of the sample: (a) $0.2 \mathrm{THz}$, (b) $0.4 \mathrm{THz}$, (c) $0.6 \mathrm{THz}$, (d) $0.8 \mathrm{THz}$.

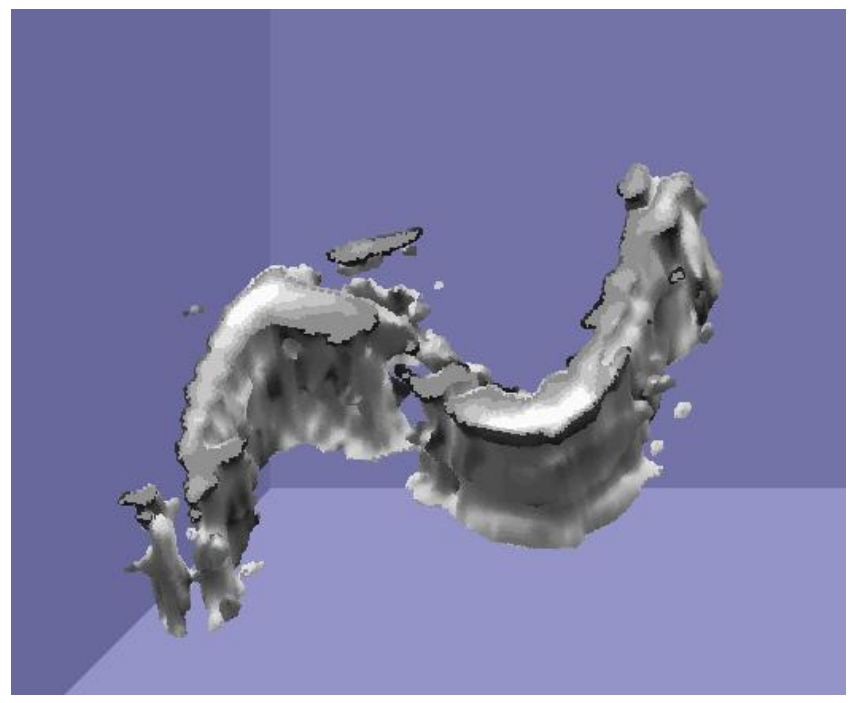

Figure 4. A 3D reconstruction of the sheet of polyethylene. The measured data was reconstructed using the filtered backprojection algorithm The phases of the THz pulses were used as the input to the algorithm. 


\subsection{T-ray DT}

T-ray DT provides a complimentary technique to T-ray CT. The propagation model in T-ray CT neglects diffraction effects. This causes difficulties in the reconstruction of some targets. The diffraction tomography system provides a speed increase of an order of magnitude over T-ray CT and allows diffraction effects to be observed and utilised during reconstruction. In T-ray DT $2 \mathrm{D}$ electro-optic sampling ${ }^{12}$ is used together with modified reconstruction algorithms to dramatically expand the capabilities of T-ray tomography. The T-ray diffraction tomography system is shown in Fig. 3. THz pulses are generated using the same laser and photoconductive antenna as for T-ray CT described earlier. The pump and probe beams are expanded to a beam diameter of $2.5 \mathrm{~cm}$ using telescope lens systems. After transmission through the sample the 2D profile of the THz pulse is measured using a large $4 \mathrm{~mm}$ thick ZnTe electro-optic crystal. The crossed polarisers reduce the background CCD intensity.

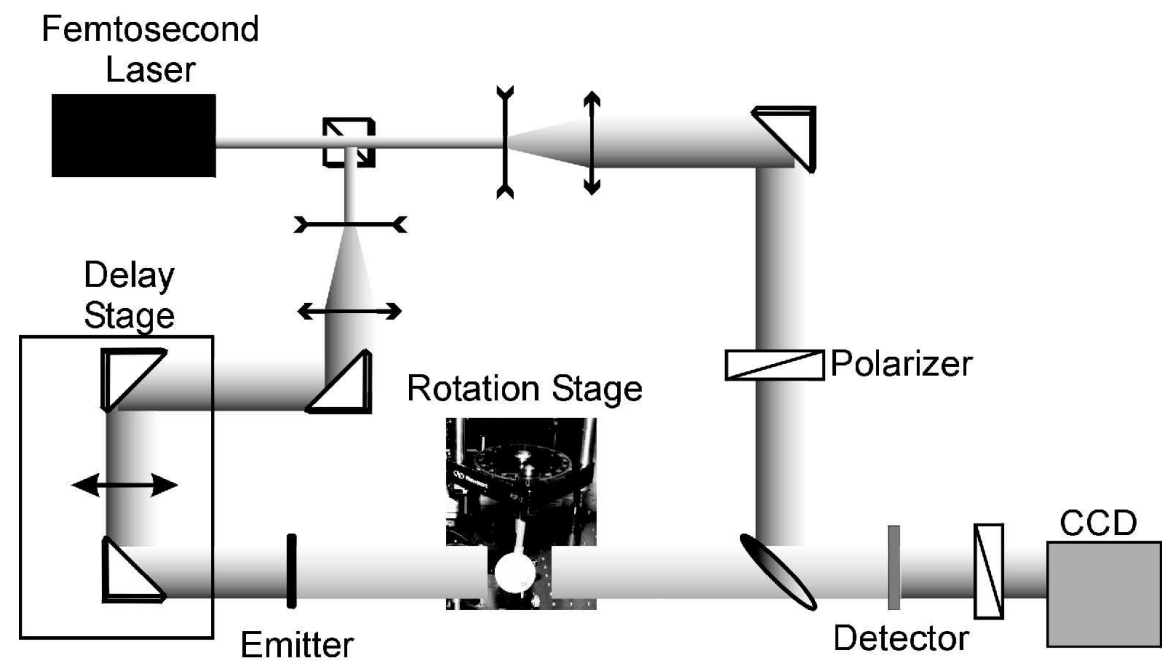

Figure 5. T-ray diffraction tomography hardware schematic. Lenses are used to expand the pump and probe beams to a diameter of $2.5 \mathrm{~cm}$. THz pulses are generated using a wide aperture photoconductive antenna and detected using $2 \mathrm{D}$ electro-optic sampling in a $4 \mathrm{~mm}$ thick $\langle 110\rangle \mathrm{ZnTe}$ detector crystal.

A single motion stage is required to scan the $\mathrm{THz}$ temporal profile and the target is rotated to obtain an image at multiple projection angles. The full data acquisition period for a CCD exposure time of $50 \mathrm{~ms}$ per frame and a projection step size of $10^{\circ}$ is approximately 8 minutes. To test this system a simple structure consisting of 3 rectangular polyethylene cylinders was imaged. This test structure is illustrated in Fig. 6 and the geometry shown in Fig. 7.

To reconstruct the T-ray DT data we rely on diffraction theory and solve a linearised form of the wave equation to recover the scattering object. A number of authors have recently described algorithms for processing the scattered or diffracted fields in pulsed terahertz radiation experiments. These experiments have considered the problem of reconstructing the 2 dimensional spatial transmission (or reflection) function of objects smaller than the THz beam size using measurements of the diffracted $\mathrm{THz}$ field at several off axis angles. These techniques have used time-reversal of the Huygens-Fresnel diffraction formula ${ }^{13,14}$ and Kirchhoff migration. ${ }^{5}$ Both of these experiments require sequential measurement of the diffracted field at multiple locations by repositioning the detector. By using 2D electro-optic sampling we achieve a much faster acquisition rate, however SNR and field of view are significantly reduced. By rotating the target we are able to achieve reliable reconstruction of 3D targets.

Our method is based on the field of diffraction tomography. ${ }^{15,16}$ These methods are only applicable to weakly scattering objects so we restrict the test samples to this case. In the future non-linear iterative techniques will enable more general targets to be reconstructed. 


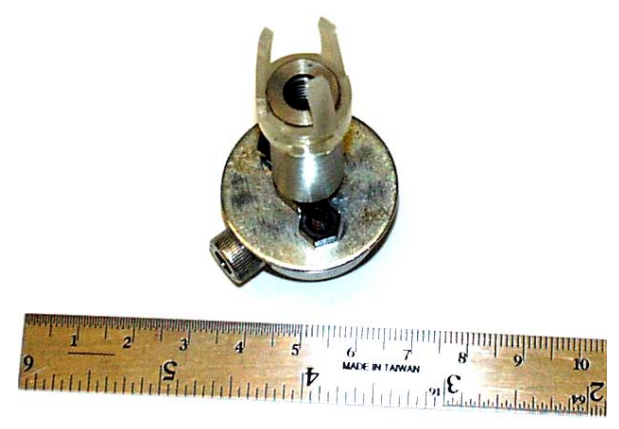

Figure 6. The test structure imaged by the Tray DT system. The target consists of 3 rectangular polyethylene cylinders.

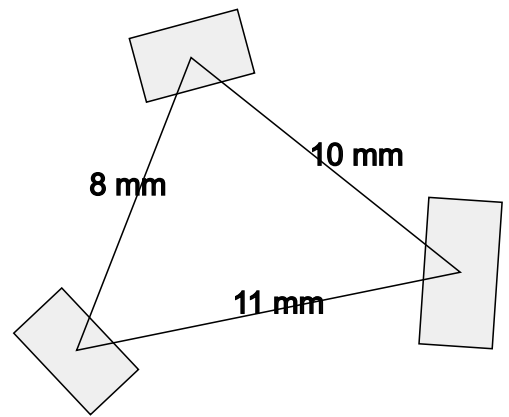

Figure 7. The geometry of the T-ray DT test structure. The rectangular cylinders have dimensions of $2.0 \times 1.5,3.5 \times 1.5$ and $2.5 \times 1.5$ (clockwise from top)

We linearise the scalar Helmholtz equation:

$$
\left(\nabla^{2}+k_{0}^{2}\right) u_{s}(\mathbf{r})=-\mathbf{u}(\mathbf{r}) \mathbf{o}(\mathbf{r}),
$$

where the field $u(\mathbf{r})$ is the sum of the incident, $u_{0}(\mathbf{r})$ and scattered $u_{s}(\mathbf{r})$ components, $o(\mathbf{r})$ is the object function of interest and $k_{0}$ is the wavenumber of the background medium. We deal with the reconstruction at a single frequency, which is achieved by Fourier transforming the received pulses and using the Fourier coefficients at a single frequency. A solution to this equation can be obtained in terms of the Green's function $g\left(\mathbf{r}-\mathbf{r}^{\prime}\right)$,

$$
u_{s}(\mathbf{r})=\int \mathbf{g}\left(\mathbf{r}-\mathbf{r}^{\prime}\right) \mathbf{o}\left(\mathbf{r}^{\prime}\right) \mathbf{u}\left(\mathbf{r}^{\prime}\right) \mathbf{d} \mathbf{r}^{\prime} .
$$

Two common linearisations of this equation are obtained using the Born and Rytov approximations. Here we adopt the Rytov approximation as it is generally less restrictive, especially at shorter wavelengths. This approximation and the required assumptions are described in a number of texts. ${ }^{15,17}$ The resulting equation is

$$
u_{0} \phi_{s}=\int g\left(\mathbf{r}-\mathbf{r}^{\prime}\right) \mathbf{o}\left(\mathbf{r}^{\prime}\right) \mathbf{u}_{\mathbf{0}}\left(\mathbf{r}^{\prime}\right) \mathbf{d} \mathbf{r}^{\prime},
$$

where $\phi_{s}$ is the scattered phase.

The Fourier diffraction theorem is then used to obtain the object function from our measurements of the incident field and scattered phase. The Fourier diffraction theorem states that the spatial Fourier transform of the scattered field along the receiver line, $U_{s}(w)$ is proportional to the $2 \mathrm{D}$ spatial Fourier transform of the object function along a semi-circular arc, $O\left(w, \sqrt{k_{0}^{2}-w^{2}}-k_{0}\right)$. Bilinear interpolation is then used to estimate the values of $O(u, v)$ on a regular grid, and the inverse Fourier transform then yields the object function in 2D space. The object function is related to the target's complex refractive index by the equation

$$
o(\mathbf{r})=\mathbf{k}_{\mathbf{0}}^{2}\left[\hat{\mathbf{n}}^{2}(\mathbf{r})-\mathbf{1}\right] .
$$

The results of this reconstruction for the target shown in Fig. 6 is shown in Fig. 8. The reconstruction was performed using a frequency of $0.1 \mathrm{THz}$, which provides the maximum signal to noise ratio (SNR) for our antenna source. The scattered fields at each height were averaged to provide a high fidelity reconstruction. Alternatively each slice may be reconstructed individually and combined to form a 3D image. This is illustrated in Fig. 9. 


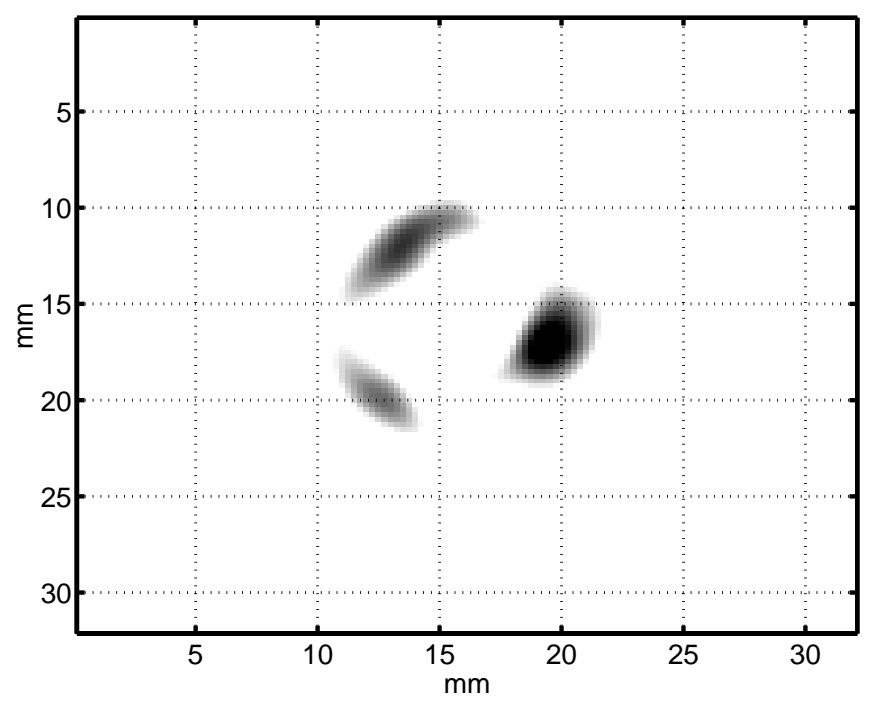

Figure 8: Reconstructed cross-section of the polyethylene cylinders. The three cylinders are clearly differentiated.

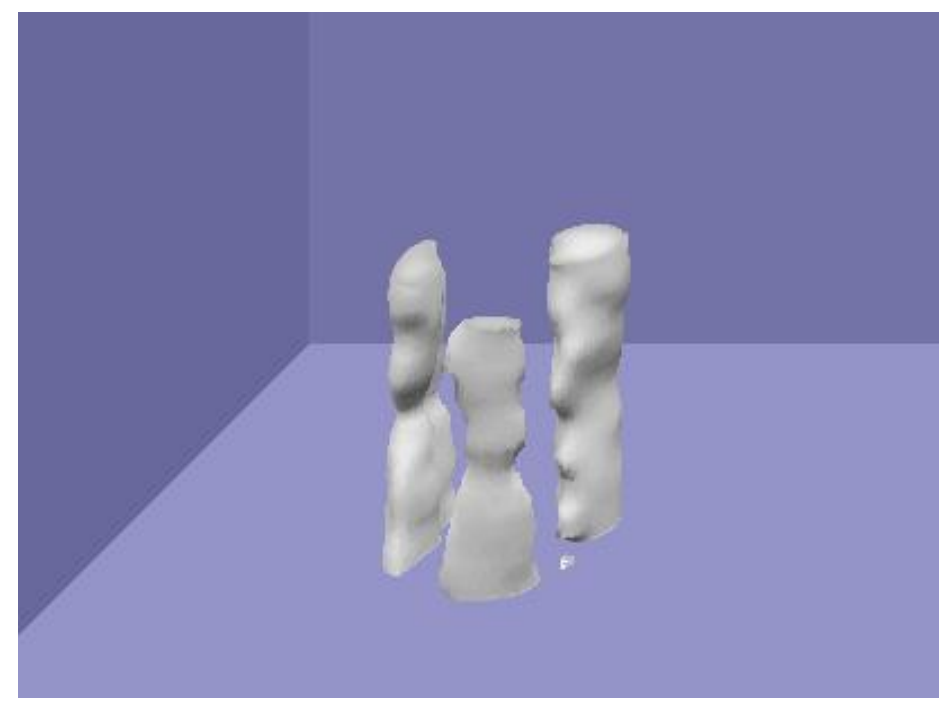

Figure 9. Reconstructed 3D image of the polyethylene cylinders. Each horizontal slice was reconstructed independently and combined to form a 3D image. The visible ripples on the surface of the cylinders are caused by noise. 


\subsection{Fresnel Lens Tomography}

The focal length of a Fresnel lens is linearly proportional to frequency. This unique property allows tomographic imaging of a target using multiple frequencies. Using a Fresnel lens at different frequencies of the imaging beam, we are able to image the objects at various positions along the beam propagation path onto the same imaging plane. This procedure enables the reconstruction of an object's tomographic contrast image by assembling the frequency-dependent images, providing a new tomographic imaging modality. A binary lens is a Fresnel zone plate with phase or amplitude modulated patterns, formed by a series of concentric ring structures. The main focal length is defined as ${ }^{18}$

$$
f_{v}=\frac{r_{p}^{2}}{2 \lambda}=\frac{r_{p}^{2}}{2 c} v \propto v
$$

where $r_{p}^{2}$ is the Fresnel zone period with a dimension of area, $\lambda$ is the wavelength, $c$ is the speed of light, and $n$ is the frequency. The focal length $f_{v}$ is linearly proportional to frequency $v$. For a single-lens imaging system, under the paraxial ray approximation, the relationship between object distance $z$, image distance $z^{\prime}$ and the focal length $f_{v}$ is governed by the imaging equation,

$$
\frac{1}{z}+\frac{1}{z^{\prime}}=\frac{1}{f_{v}}
$$

with the magnification factor of $\left(-z^{\prime} / z\right)$. If the image plane position is fixed, and therefore $z^{\prime}$ is fixed, for a wave with frequency $v$, due to the frequency dependent focal length $f_{v}$, the object distance $z$ is also frequency dependent. Combining Eqn. 8 and 9, z yields,

$$
z=\frac{f_{v} z^{\prime}}{z^{\prime}-f_{v}}=\frac{r_{p}^{2} z^{\prime} v}{2 c z^{\prime}-r_{p}^{2} v} .
$$

At each frequency $v$, there is a corresponding value of $z$, and a target at this position $z$ can be well imaged onto the position $z^{\prime}$ at the imaging plane. In order to keep the imaging distance $z>0$, it requires $z^{\prime}-f_{v}>0$, therefore, $v<2 c z^{\prime} / r_{p}^{2}$ for each fixed $z^{\prime}$.

The experimental setup of an imaging system with a CCD camera was similar to the one used for the characterization of a $\mathrm{THz}$ wave reported elsewhere. ${ }^{19}$ A $30-\mathrm{mm}$ diameter silicon binary lens with a focal length of $2.5 \mathrm{~cm}$ at $1 \mathrm{THz}$ was used as the $\mathrm{THz}$ wave Fresnel lens. By scanning the time delay between the THz and optical probe beams, a temporal waveform of the $\mathrm{THz}$ wave at each pixel on the image plane was measured using a CCD camera. Fourier transformation of the temporal waveforms provided the $\mathrm{THz}$ field amplitude (or intensity) distribution on the image plane at each frequency. The measured two-dimensional $\mathrm{THz}$ field distribution at each frequency formed the image of $\mathrm{THz}$ field transmission of a target at each corresponding position along the $z$-axis.

Figure 10 schematically illustrates the tomographic imaging arrangement. Three plastic sheets with different patterns were placed along the $\mathrm{THz}$ beam path, and their distances to the lens, corresponding to z in Eqn. 10 were $3 \mathrm{~cm}, 7 \mathrm{~cm}$ and $14 \mathrm{~cm}$, respectively. Inverted images of patterns on the sensor plane at distance $z^{\prime}=6$ $\mathrm{cm}$ were measured at frequencies of $0.74 \mathrm{THz}, 1.24 \mathrm{THz}$, and $1.57 \mathrm{THz}$, respectively. At each frequency, the Fresnel lens imaged a different plane section of a target object corresponding to a certain depth while images from other depths remained blurred. Each point in the different object planes along the $z$-axis was mapped onto a corresponding point on the $z^{\prime}$ plane (sensor plane) with the magnification factor $-z^{\prime} / z$ at their corresponding frequencies. The calculated frequency-dependent magnification factor is defined as $-z^{\prime} / z$. The ratio of the measured image size to the actual size of the pattern agrees well with the calculated magnification factor.

\section{DISCUSSION}

\subsection{Comparison of the Techniques}

The three tomography techniques each have advantages and disadvantages. T-ray CT is very attractive due to its ability to extract the frequency dependent refractive index at each pixel of a 3D image. The focused THz beam allows a higher SNR to be achieved than either of the other techniques. However, this technique has a number of important problems. It is very time consuming; a typical image of size $100 \times 100$ pixels measured at 18 projection 


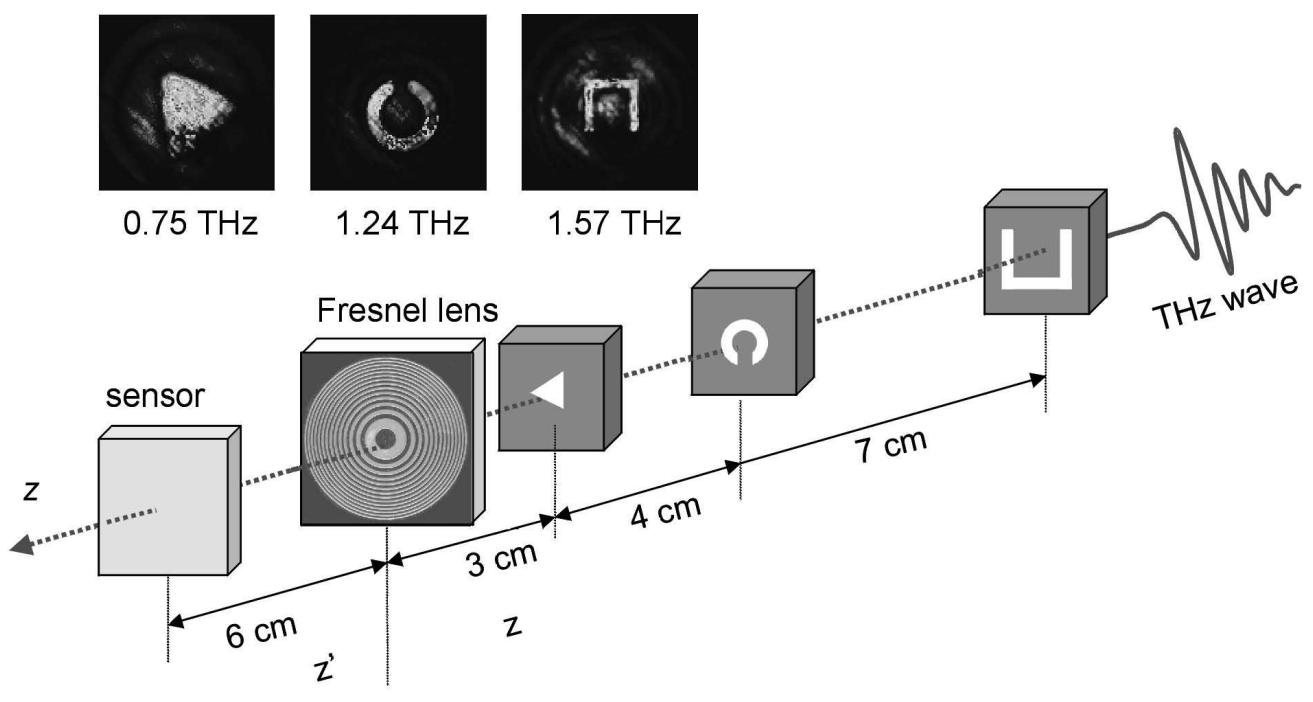

Figure 10. Schematic illustration of tomographic imaging with a Fresnel lens. Targets at various locations along the beam propagation path are uniquely imaged on the same imaging sensor plane with different frequencies of the imaging beam. Three plastic sheets were cut with different patterns placed $3 \mathrm{~cm}, 7 \mathrm{~cm}$, and $14 \mathrm{~cm}$ away from the Fresnel lens. The multiple patterns are imaged on the sensor at a distance of $6 \mathrm{~cm}$ from the Fresnel lens, with inverted tomography images of the patterns at the frequencies of $0.75 \mathrm{THz}, 1.24 \mathrm{THz}$ and $1.57 \mathrm{THz}$, respectively. The measured image size is determined by the frequency dependent magnification factor, which is defined as $z^{\prime} / z$.

angles can take over an hour. As higher power THz sources become available, the SNR of the detected THz will increase and the CCD acquisition time can be reduced. The fundamental limit using our current hardware is an acquisition time of 15 minutes for the size image described previously. Another important problem is that of diffraction. T-ray CT works well for targets with features that are large relative to the wavelength of the THz radiation $(0.3 \mathrm{~mm}$ at $1 \mathrm{THz})$, however for more complex targets with fine structure the filtered back-projection algorithm is unable to accurately reconstruct the target because diffraction effects dominate the measurements. The focal width and depth of the T-ray beam are also important parameters which impact on the resolution and accuracy of T-ray CT.

T-ray DT overcomes many of the problems of T-ray CT. It can be performed relatively quickly by aid of 2D $\mathrm{THz}$ imaging, and the diffracted field is explicitly used in the reconstruction. As the diffracted field is detected in the mid/near field there is the potential to perform the reconstruction with sub-wavelength resolution. The major difficulty in T-ray DT is the reconstruction algorithm. The Rytov approximation is only valid for small, low refractive index targets. However, much work has been performed inverting the wave equation for tomographic reconstruction in ultrasound and microwave fields and it is expected that variations of these algorithms will prove fruitful for T-ray DT. The currently implemented T-ray DT system is only suitable for targets smaller than the $\mathrm{THz}$ sensor, which has a diameter of $2 \mathrm{~cm}$. However, future systems will use a telescope arrangement of $\mathrm{THz}$ polyethylene lens to allow larger targets to be imaged. A further difficulty of T-ray DT is the low SNR caused by spreading the limited $\mathrm{THz}$ power (approximately $4 \mu \mathrm{W}$ average power) over the entire sensor area. T-ray DT systems will benefit greatly from higher power $\mathrm{THz}$ sources.

A Fresnel lens allows tomographic images to be obtained without requiring rotation of the target. Using a single projection image, the $\mathrm{THz}$ spectral data provides sufficient information to reconstruct the full 3D target. Although this method does not provide spectroscopic information it has the potential to acquire 3D images of targets extremely quickly. The depth of focus of the THz wave introduces a depth uncertainty. Since the depth uncertainty of the target position is equal to the depth of focus divided by the square of the magnification factor, ${ }^{20}$ the uncertainty of the target position is also a function of $z$. The measured depth of focus in the imaging system is $3 \mathrm{~mm}$. For a large value of $z\left(z>>z^{\prime}\right)$, the depth resolution decreases. Although this demonstration uses a 
broadband $\mathrm{THz}$ radiation as the imaging beam, the Fresnel lens tomographic imaging concept is also applicable to a tunable narrowband imaging beam, and can be applied to other frequency ranges, including the visible.

\section{CONCLUSION}

We have developed and demonstrated three T-ray tomography systems. These systems each have important advantages that lend them to specific applications. Applications of these techniques may include 3-dimensional biological tomographic imaging include functional imaging of cancerous tumors, mapping blood flow and oxidation, or fat content of tissues. Other applications are non-destructive signature detection of biological materials such as anthrax and TNT for mail, package and luggage screening.

\section{ACKNOWLEDGMENTS}

We are grateful to Mr. Edward Walsby of the University of Canterbury, New Zealand for providing the silicon binary lens, and acknowledge helpful discussions with Samuel Mickan.

This work was supported by the Australian Research Council, the U.S. National Science Foundation and the U.S. Army Research Office.

Bradley Ferguson would like to thank the Australian-American Fulbright Commission.

\section{REFERENCES}

1. D. M. Mittleman, S. Hunsche, L. Boivin, and M. C. Nuss, "T-ray tomography," Optics Letters 22(12), pp. 904-906, 1997.

2. B. Ferguson, S. Wang, D. Gray, D. Abbott, and X.-C. Zhang, "T-ray computed tomography," Optics Letters (in press), 2002.

3. B. Ferguson, S. Wang, D. Gray, D. Abbott, and X.-C. Zhang, "T-ray diffraction tomography," in OSA Trends in Optics and Photonics (TOPS), The Thirteenth International Conference on Ultrafast Phenomena, 72, pp. 450-451, Optical Society of America, (Washington, DC.), 2002.

4. S. Wang and X.-C. Zhang, "Tomographic imaging with a terahertz wave Fresnel lens," Optics Letters (submitted), 2002.

5. T. D. Dorney, J. L. Johnson, J. V. Rudd, R. G. Baraniuk, W. W. Symes, and D. M. Mittleman, "Terahertz reflection imaging using Kirchhoff migration," Optics Letters 26(19), pp. 1513-1515, 2001.

6. B. B. Hu and M. C. Nuss, "Imaging with terahertz waves," Optics Letters 20(16), pp. 1716-1718, 1995.

7. M. van Exter and D. Grischkowsky, "Optical and electronic properties of doped silicon from 0.1 to $2 \mathrm{THz}$," Applied Physics Letters 56(17), pp. 1694-1696, 1990.

8. R. H. Jacobsen, D. M. Mittleman, and M. C. Nuss, "Chemical recognition of gases and gas mixtures with terahertz waves," Optics Letters 21(24), pp. 2011-2013, 1996.

9. M. Nagel, P. Haring Bolivar, M. Brucherseifer, and H. Kurz, "Integrated THz technology for label-free genetic diagnostics," Applied Physics Letters 80(1), pp. 154-156, 2002.

10. Z. Jiang and X.-C. Zhang, "Electro-optic measurement of THz field pulses with a chirped optical beam," Applied Physics Letters 72(16), pp. 1945-1947, 1998.

11. G. T. Herman, Image Reconstruction From Projections - The Fundamentals of Computerized Tomography, Academic Press, New York, 1980.

12. Q. Wu, T. D. Hewitt, and X.-C. Zhang, "Two-dimensional electro-optic imaging of terahertz beams," Applied Physics Letters 69(8), pp. 1026-1028, 1996.

13. A. B. Ruffin, J. Decker, L. Sanchez-Palencia, L. Le Hors, J. F. Whitaker, T. B. Norris, and J. V. Rudd, "Time reversal and object reconstruction with single-cycle pulses," Optics Letters 26(10), pp. 681-683, 2001.

14. A. B. Ruffin, J. Van Rudd, J. Decker, L. Sanchez-Palencia, L. Le Hors, J. F. Whitaker, and T. B. Norris, "Time reversal terahertz imaging," IEEE Journal of Quantum Electronics 38(8), pp. 1110-1119, 2002.

15. A. C. Kak and M. Slaney, Principles of Computerized Tomographic Imaging, Society of Industrial and Applied Mathematics, 2001. 
16. A. J. Devaney, "A filtered backpropagation algorithm for diffraction tomography," Ultrasonic Imaging 4, pp. 336-350, 1982.

17. F. Natterer and F. Wübbeling, Mathematical Methods in Image Reconstruction, Society of Industrial and Applied Mathematics, Philadelphia, U.S.A., 2001.

18. J. Jahns and S. J. Walker, "Two-dimensional array of diffractive microlenses fabricated by thin film deposition," Applied Optics 29(7), pp. 931-936, 1990.

19. S. Wang, T. Yuan, E. D. Walsby, R. J. Blaikie, S. M. Durbin, D. R. S. Cumming, J. Xu, and X.-C. Zhang, "Characterization of T-ray binary lenses," Optics Letters 27(13), pp. 1183-1185, 2002.

20. B. E. A. Saleh and M. C. Teich, Fundamentals of Photonics, John Wiley \& Sons, Inc., New York, 1991. 